\title{
Global simulation of proton precipitation due to field line curvature during substorms
}

\author{
M. L. Gilson, ${ }^{1}$ J. Raeder, ${ }^{1}$ E. Donovan, ${ }^{2}$ Y. S. Ge, ${ }^{1}$ and L. Kepko ${ }^{3}$ \\ Received 25 January 2012; revised 21 March 2012; accepted 21 March 2012; published 9 May 2012.
}

[1] The low latitude boundary of the proton aurora (known as the Isotropy Boundary or IB) marks an important boundary between empty and full downgoing loss cones.

There is significant evidence that the IB maps to a region in the magnetosphere where the ion gyroradius becomes comparable to the local field line curvature. However, the location of the IB in the magnetosphere remains in question. In this paper, we show simulated proton precipitation derived from the Field Line Curvature (FLC) model of proton scattering and a global magnetohydrodynamic simulation during two substorms. The simulated proton precipitation drifts equatorward during the growth phase, intensifies at onset and reproduces the azimuthal splitting published in previous studies. In the simulation, the pre-onset IB maps to $7-8 \mathrm{R}_{\mathrm{E}}$ for the substorms presented and the azimuthal splitting is caused by the development of the substorm current wedge.

The simulation also demonstrates that the central plasma sheet temperature can significantly influence when and where the azimuthal splitting takes place.

Citation: Gilson, M. L., J. Raeder, E. Donovan, Y. S. Ge, and L. Kepko (2012), Global simulation of proton precipitation due to field line curvature during substorms, J. Geophys. Res., 117, A05216, doi:10.1029/2012JA017562.

\section{Introduction}

[2] Much effort has been spent to determine the Earth's magnetic field topology under various inputs from the solar wind. This information is useful because it can be used to draw connections between auroral features and magnetospheric phenomena. In a recent example of this, Nishimura et al. [2010] was able to correlate individual patches of patchy aurora with chorus waves in the magnetosphere. In other recent papers, authors have speculated about the origin of the wavy structure that appears along the substorm onset arc a few seconds before onset [e.g., Raeder et al., 2010]. For substorms, much debate has existed about where the breakup arc originates compared to the location of the nearearth reconnection. Since the mapping is uncertain, this remains a fundamental disagreement between researchers.

[3] One of the difficulties associated with mapping auroral features during substorms arises because the magnetotail is highly stretched and can globally reconfigure over short periods of time. Because typical empirical models [Tsyganenko, 1989, 2002a, 2002b] are parameterized by geomagnetic indices and Interplanetary Magnetic Field (IMF) measurements

\footnotetext{
${ }^{1}$ Space Science Center, University of New Hampshire, Durham, New Hampshire, USA.

${ }^{2}$ Department of Physics and Astronomy, University of Calgary, Calgary, Alberta, Canada.

${ }^{3}$ NASA Goddard Space Flight Center, Greenbelt, Maryland, USA.

Corresponding author: M. L. Gilson, Space Science Center, University of New Hampshire, 8 College Rd., Durham, NH 03824, USA.

(mgilson@artemis.sr.unh.edu)

Copyright 2012 by the American Geophysical Union. 0148-0227/12/2012JA017562
}

$\left(\mathrm{K}_{\mathrm{P}}\right.$, DST, $\mathrm{AE}, \mathrm{B}_{\mathrm{z}}$, etc.) they only represent an average field configuration and are therefore most applicable to general statistical studies as opposed to particular events. To circumvent this problem, authors often use data from low-altitude satellites in the auroral oval to learn about particle distributions and field structure in the source regions [e.g., Sergeev et al., 1993, 2012].

[4] One of the more promising routine measurements made by low altitude satellites is the location of the isotropic boundary (IB). Equatorward (earthward) of the low latitude boundary of the proton aurora, low altitude satellites measure double loss cone ion distributions indicating bouncetrapped particles. However, in measurements at altitudes above the proton auroral regions, satellites typically measure a full downgoing loss cone [Donovan et al., 2003a]. The IB is the location of the transition between the full and empty downgoing loss cones. The particles in the downgoing loss cone have energies consistent with central plasma sheet (CPS) ions [Eather, 1967] and it is now commonly accepted that they are generated by scattering in the CPS. Generally, the IB is a function of particle energy and species, however, it generally corresponds well with b2i boundary of Newell et al. [1996], defined as the location of the maximum of the integrated ion energy flux.

[5] The IB and the b2i have a long history of being used to infer the degree of magnetic field stretching in the inner magnetotail. Simulations have shown that in regions where the field line curvature (FLC) becomes comparable to the particle gyroradius there is significant pitch angle scattering [Sergeev and Tsyganenko, 1982]. The criterion for strong enough scattering to fill the loss cone found by Sergeev et al. [1983] is that the magnetic field radius of curvature divided by the particle gyroradius is less than 8 . It is now more 
common to see the $\kappa$-parameter [Büchner and Zelenyi, 1987]. In this form, the strong scattering criterion is:

$$
\kappa=\sqrt{\frac{R_{c}}{\rho}} \leq \sqrt{8}
$$

where $R_{c}$ is the magnetic field radius of curvature and $\rho$ is the particle gyroradius.

[6] If the scattering is caused by FLC, then the IB maps to an important transition from moderately stretched to highly stretched field lines in the magnetosphere. This inspired Sergeev et al. [1993] and Newell et al. [1998] to show that the magnetic field inclination at geosynchronous orbit is highly correlated with the IB and b2i respectively on the midnight meridian. They used the auroral boundaries to add an additional constraints on the existing empirical mapping models. The correlation was good enough $(\mathrm{r} \sim 0.9)$ to lead Gvozdevsky and Sergeev [1996] to define a magnetotail (MT) index based on the IB latitude. More recently, Meurant et al. [2007] showed that the IB remains well correlated with the extent of magnetic field stretching even during active times like the substorm expansion phase. They also showed that the stretching typically decreases first in the dawn sector.

[7] In addition to correlating with the degree of stretching in the magnetotail, the onset arc typically sits near the poleward boundary of the proton precipitation [Lessard et al., 2007; Deehr and Lummerzheim, 2001]. Thus, the onset arc definitely maps tailward of the IB during substorms. As much debate revolves around the timing of the auroral brightening compared to the transit time of observed substorm signatures in the tail, the location of the IB could at least place a lower bound on the mapping of the onset location. Synoptic descriptions of how the electron and proton auroral precipitation boundaries are positioned relative to one another as a function of magnetic local time have been presented in Creutzberg et al. [1988] and Mende [2003].

[8] Because the importance of the IB and the success of the FLC model, observations of the IB and b2i have also been inferred from meridian scanning photometers (MSP) [Donovan et al., 2003b] and from global images taken by the SI-12 instrument on the IMAGE spacecraft [Blockx et al., 2005; Meurant et al., 2007]. However, despite the large number of observations, where the IB maps in the tail is still an open question.

[9] Recently, Gilson et al. [2011] focused further on the longitudinal evolution the proton aurora during substorms and showed that the proton aurora commonly splits after the onset with eastward and westward traveling precipitation fronts. The splitting is more likely to occur for substorms with lower AL. Based on the FLC model, they speculated that the split region corresponds to the edges of the substorm current wedge (SCW) as the field curvature inside the SCW should be much larger than outside. However, in that study, it was unclear why the proton aurora should split during some events and not others. One hypothesis was that the scattering could continue in regions with a quasi-dipolarized magnetic field geometry if the plasma temperature was significantly high. This hypothesis was supported by the observation that weaker substorms are less likely to split than their stronger counterparts because the weaker substorms are probably associated with less intense dipolarization. Alternatively, wave-particle interactions [Ashour-Abdalla and Thorne, 1978] could be responsible for scattering particles into the loss cone in the SCW.

[10] A study by Donovan et al. [2003a] showed that the energy dependence of the IB latitude is rarely completely consistent with what is expected from the FLC model. It is reasonable to expect that higher energy particles will scatter in a less stretched field due to their larger average gyroradii. This implies that the IB for high energy particles should be observed at lower latitudes than the IB of low energy particles to be consistent with the FLC model. Of approximately 1000 FAST passes through the auroral oval, only $\sim 10 \%$ of the transits had energy orderings completely consistent with the FLC model while $\sim 10 \%$ were completely inconsistent. The discrepancy could be due to wave-particle interactions. If that turns out to be the case, using the proton aurora to mark the low $\kappa$ boundary may not valid. However, it is also possible that some other physical process is occurring at the inner edge of the CPS to reverse the ordering. One candidate is charge exchange leading to precipitating heavier ions [Kistler et al., 1998] since the FAST-ESA instrument used in the Donovan et al. [2003a] study did not have the mass resolution to separate out that effect.

[11] In this study, we use the FLC model with the OpenGGCM global MHD code to simulate the proton precipitation during substorm conditions using realistic solar wind input. We will present two substorms. The first is the March 23, 2007 "THEMIS First Light" substorm analyzed by Angelopoulos et al. [2008], Raeder et al. [2008], Runov et al. [2008], Keiling et al. [2008], Lessard et al. [2009] and others. The second is a substorm observed by the IMAGE Far Ultraviolet (FUV) camera [Mende et al., 2000b, 2000a, 2000c] on April 28, 2001. In the simulations, the proton precipitation splits longitudinally and forms westward and eastward traveling fronts similar to the description given by Gilson et al. [2011]. This splitting is well correlated with the longitudinal growth of the SCW in the simulation. Similar splitting is seen by the IMAGE FUV camera for the April 28, 2001 substorm. Therefore, we show that the FLC model is sufficient to explain the observed proton auroral splitting. We also show that for some conditions the precipitation due to FLC can exist in a quasi-dipolarized SCW which eliminates the need to consider wave particle interactions to explain the lack of proton auroral splitting during weaker substorms.

\section{OpenGGCM}

[12] To address the mapping problem, we are using OpenGGCM for this study. OpenGGCM is a global simulation which solves the MHD equations on a stretched Cartesian grid. The advantage of this grid structure is that the resolution is able to be increased in areas where it is needed (e.g., the magnetotail) while keeping the total number of cells manageable. The main advantage of using a global model for this study is that auroral features can be mapped to structures in the tail self-consistently without needing to wait for a fortunate conjunction between satellites and/or ground observations on a clear day. Also, in some cases, the simulation mapping can explain observed data better than available empirical models [Angelopoulos et al., 2008]. 


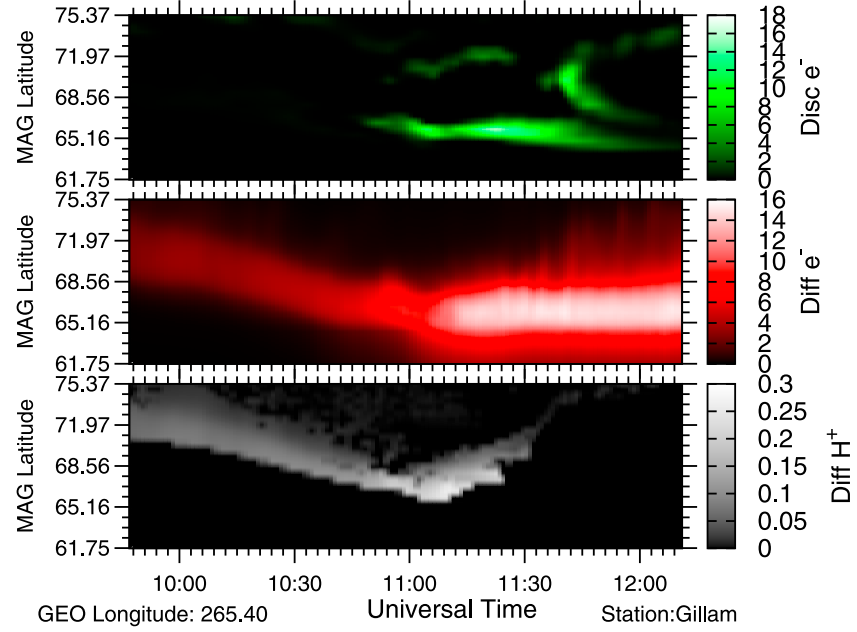

Figure 1. Synthetic keogram along the Gillam meridian for the March 23, 2007 simulation. (top) The electron precipitation accelerated in regions of upward field aligned current, (middle) electron precipitation calculated from the thermal electron flux, and (bottom) the proton precipitation calculated as described in the text. The equatorward drifting and intensity increase near the onset is evident.

[13] The boundary conditions on the dayside face of the simulation domain are set by data from a solar wind monitor and the other five faces have open boundary conditions. The three dimensional structure of the solar wind data is inferred by the procedure described in Raeder et al. [2001, section 3] using the minimum variance analysis of Sonnerup and Cahill [1967]. Hereafter, that procedure is referred to as MINVAR. At times, the solar wind may not be ordered in simple sheets and so the MINVAR procedure may not accurately reproduce the field. In those cases, if the $B_{x}$ component is not varying much, setting $B_{x}$ to the average value of the interval can be a reasonable approach. In other situations, more complicated methods may be used to obtain the dayside boundary condition [e.g., Russell et al., 2000].

[14] The inner boundary is calculated by mapping simulation current and electric field to the ionosphere and using empirical models to calculate the electron precipitation parameters and potential drop. The simulation calculates the thermal electron flux from the inner magnetosphere due to scattering and also energy flux from electrons accelerated in regions of upward field aligned current. The second population likely corresponds to what is commonly referred to as discrete aurora whereas the first corresponds to diffuse aurora. The CTIM (Coupled Thermosphere-Ionosphere Model) module uses these parameters to compute the ionospheric conductances which are then used to solve for the ionospheric potential. See Fuller-Rowell et al. [1996] for more the details of CTIM and Raeder et al. [2001] for details on the coupling between OpenGGCM and CTIM. For more information on OpenGGCM, see Raeder [2003] and Raeder et al. [2008].

\section{Method}

[15] To calculate the precipitating proton energy flux in OpenGGCM, field lines are integrated using a Runge-Kutta algorithm with foot points on the ionospheric grid. The typical resolution for the ionospheric grid is half a degree in latitude and three degrees in longitude. If the field line is closed, the average particle gyroradius $(\rho)$ and field radius of curvature $\left(R_{c}\right)$ (and therefore $\kappa$ ) are calculated in the CPS from the MHD variables assuming a simple Maxwellian particle distribution. In other words,

$$
\rho=\frac{\sqrt{2 m_{i} k_{b} T_{i}}}{e|B|}
$$

and the radius of curvature is calculated by:

$$
\frac{\hat{\mathbf{n}}}{R_{c}}=-(\hat{\mathbf{b}} \cdot \nabla) \hat{\mathbf{b}}
$$

where $T_{i}$ and $m_{i}$ are the ion temperature and mass, $k_{b}$ is the Boltzmann constant, $e$ is the fundamental unit of charge, $B$ is the magnetic field and $\hat{\mathbf{b}}$ is a unit vector in the direction of $B$. Since the loss cone is typically full and $\kappa$ is known, the diffuse precipitating energy flux is calculated using

$$
F=f(\kappa) n_{i} k_{b} T_{i} \sqrt{2 k_{b} T_{i} / \pi m_{i}}
$$

where $n_{i}$ is the ion number density. Equation (4) is the same used by Raeder et al. [2008] for diffuse electron precipitation with parameters for ions substituted and with an additional dependence on $\kappa$. For this paper, $f(\kappa)$ was taken to be a simple rectangle function:

$$
f(\kappa)=\left\{\begin{array}{l}
1: \quad 0<\kappa \leq \sqrt{8} \\
0: \quad \text { otherwise }
\end{array}\right.
$$

The simple form of $f(\kappa)$ in equation (5) was chosen because of the sharp cutoff in pitch angle diffusion reported by Sergeev et al. [1983]. Other forms of $f(\kappa)$ have been reported in the literature. For example, Delcourt and Martin [1994] and Delcourt et al. [1996] showed that in the regime where $\kappa<1$, the scattering does not efficiently move particles into the loss cone. Liu et al. [2007] used $f(\kappa) \propto \mathrm{B}_{\mathrm{z}} \exp (-0.97 \sqrt{\kappa})$ to explain the pre-onset fading of the proton aurora. However, it is unclear how well the MHD approximation resolves the field curvature (and therefore $\kappa$ ) in the highly stretched CPS as one of the assumptions of MHD is that the average gyroradius is small compared to the average length scale. Therefore, these forms have not been used for this study.

[16] For each substorm presented here, the proton precipitation is poleward of the discrete electron precipitation duskward of the onset and equatorward of the discrete electron precipitation on the dawnward side consistent with the statistical studies by Creutzberg et al. [1988] and Mende [2003]. The proton precipitation also intensifies and expands poleward in the simulation.

\section{Event 1: March 23, 2007}

[17] March 23, 2007 was one of the first substorms observed by the THEMIS probes, and therefore the aspects of this event have been studied in detail by Angelopoulos et al. [2008], Raeder et al. [2008] and many others. The latter simulated the event using the OpenGGCM code. For specific details of that simulation and analysis of some of the results, the reader is referred to Raeder et al. [2008]. The 
$\mathrm{H}+$ Aurora $\left[\mathrm{mW} / \mathrm{m}^{2}\right]$

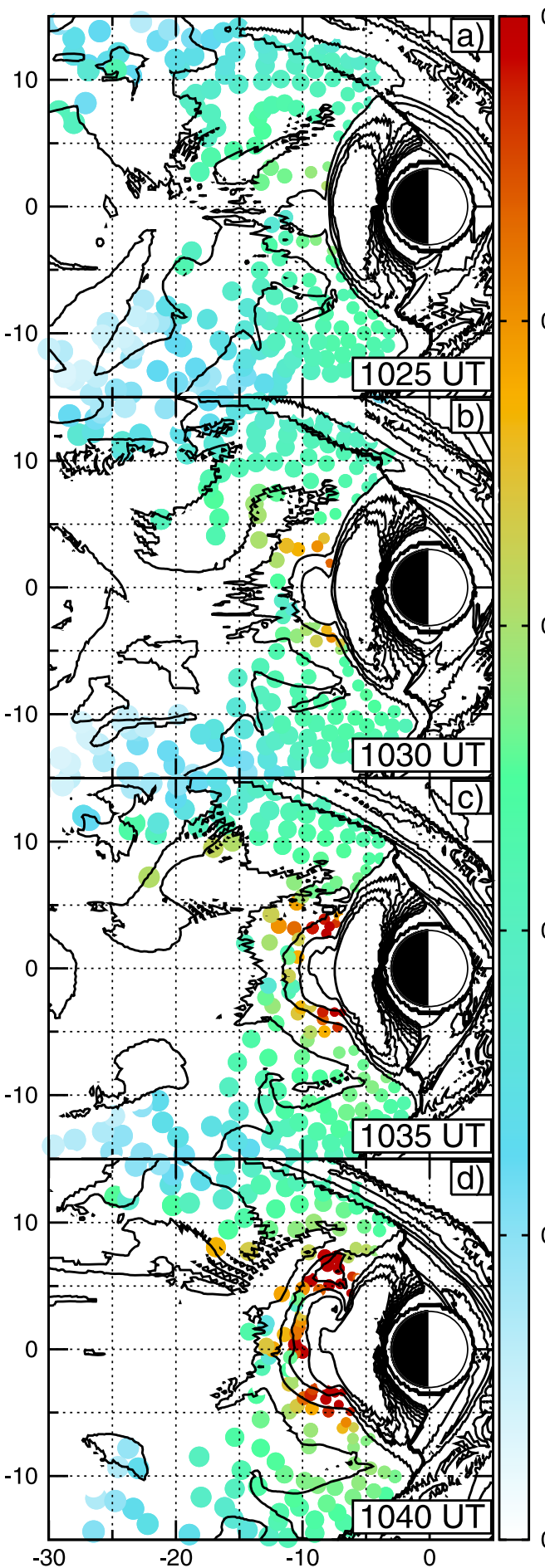

$\mathrm{B}_{\mathrm{z}}[\mathrm{nT}]$

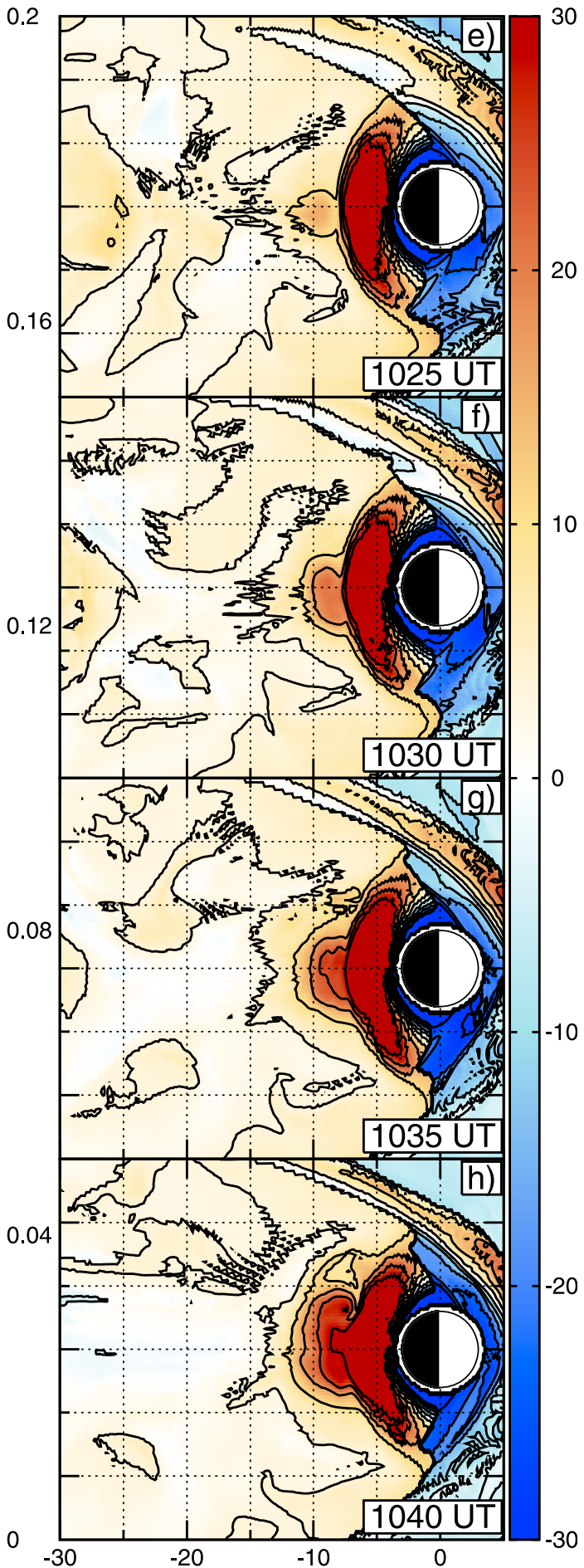

Figure 2. (a-d) Simulated proton aurora mapped along field lines to the furthest point with contours of $B_{z}$ at different times as marked. (e-h) $B_{z}$ with contours provided for reference. $B_{z}$ and contours extracted from the plane of maximum plasma $\beta$ as a proxy for the central plasma sheet.

simulation mapping agreed better with the observations than the TS01 mapping [Tsyganenko, 2002a, 2002b] because the TS01 mapped locations of the THEMIS probes lie outside the westernmost location of the auroral activation even though all the probes recorded substorm signatures (dipolarization, flows and energetic particle flux increases). A synthetic keogram of the simulated aurorae is presented in Figure 1. From the keogram, it is obvious that the simulation captures the equatorward drifting during the growth phase commonly associated with substorms. Plots from four 

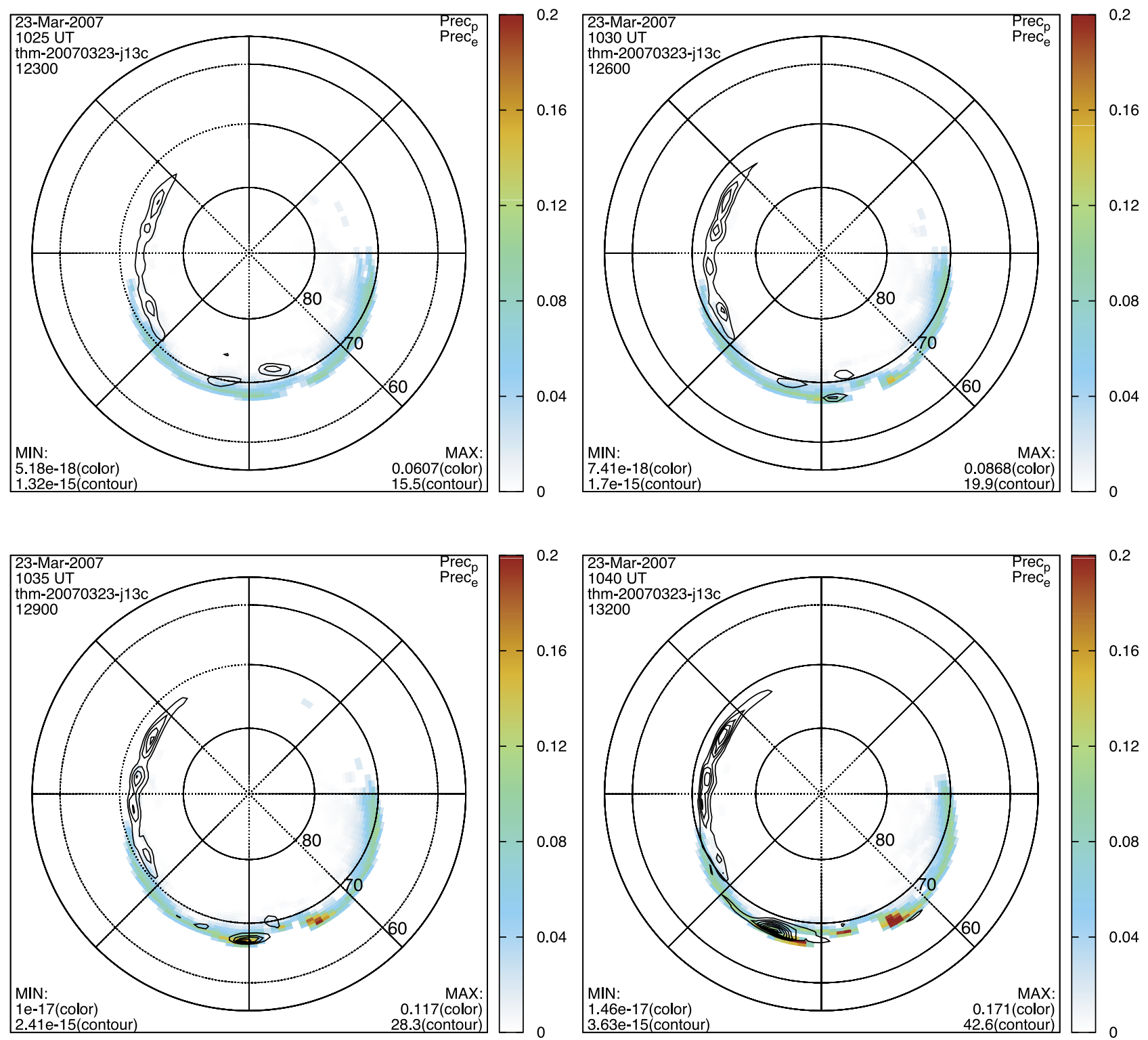

Figure 3. Simulated proton aurora (color) and discrete electron precipitation (contours) at different times in the simulation of the March 23, 2007 substorm. Local noon is at the top of each plot. The proton precipitation splits on the eastward edge of the developing westward traveling surge.

different times in the early stages of the substorm are shown in Figure 2. The first column shows the simulated proton precipitation mapped along magnetic field lines to the CPS. For the purposes of data visualization, the magnetic field is also plotted on the CPS (defined by the surface of maximum plasma $\beta$ ). The simulation shows a Dipolarization Front (DF) propagating earthward from the reconnection site at about $-15 \mathrm{R}_{\mathrm{E}}$ prior to the auroral brightening. Once the front reaches the inner magnetosphere, the flux piles up and grows toward the flanks forming the SCW (Figure 2).

[18] The proton precipitation shows the signature of the $\mathrm{SCW}$ in the ionosphere. Since the FLC model requires a highly stretched (tail-like) magnetic field or large gyroorbits (high temperature) to scatter particles into the loss cone, the dipolarized region can be seen in sudden latitudinal increases in the IB location causing a longitudinal split in the proton precipitation (compare Figures $2 \mathrm{a}-2 \mathrm{~d}$ with Figure 3 ). Another possibility is that the split is caused by the longitudinal dependence of the temperature. This would require a region of low temperature plasma at or near the onset meridian with high temperature plasma on either side. The simulation of this event shows the opposite. High temperature plasma is injected from the reconnection site into the dipolarized region and expands outward with (but inside) the current wedge.

[19] The simulation agrees with the interpretation of the longitudinal splitting of Gilson et al. [2011], however, there are unfortunately very limited ground or space proton aurora data for this event to compare with the simulation. Also, this simulation does not explain why the proton aurora splits in stronger events but not in weaker events. Because of the limited data, we use the IMAGE data set to find an event 


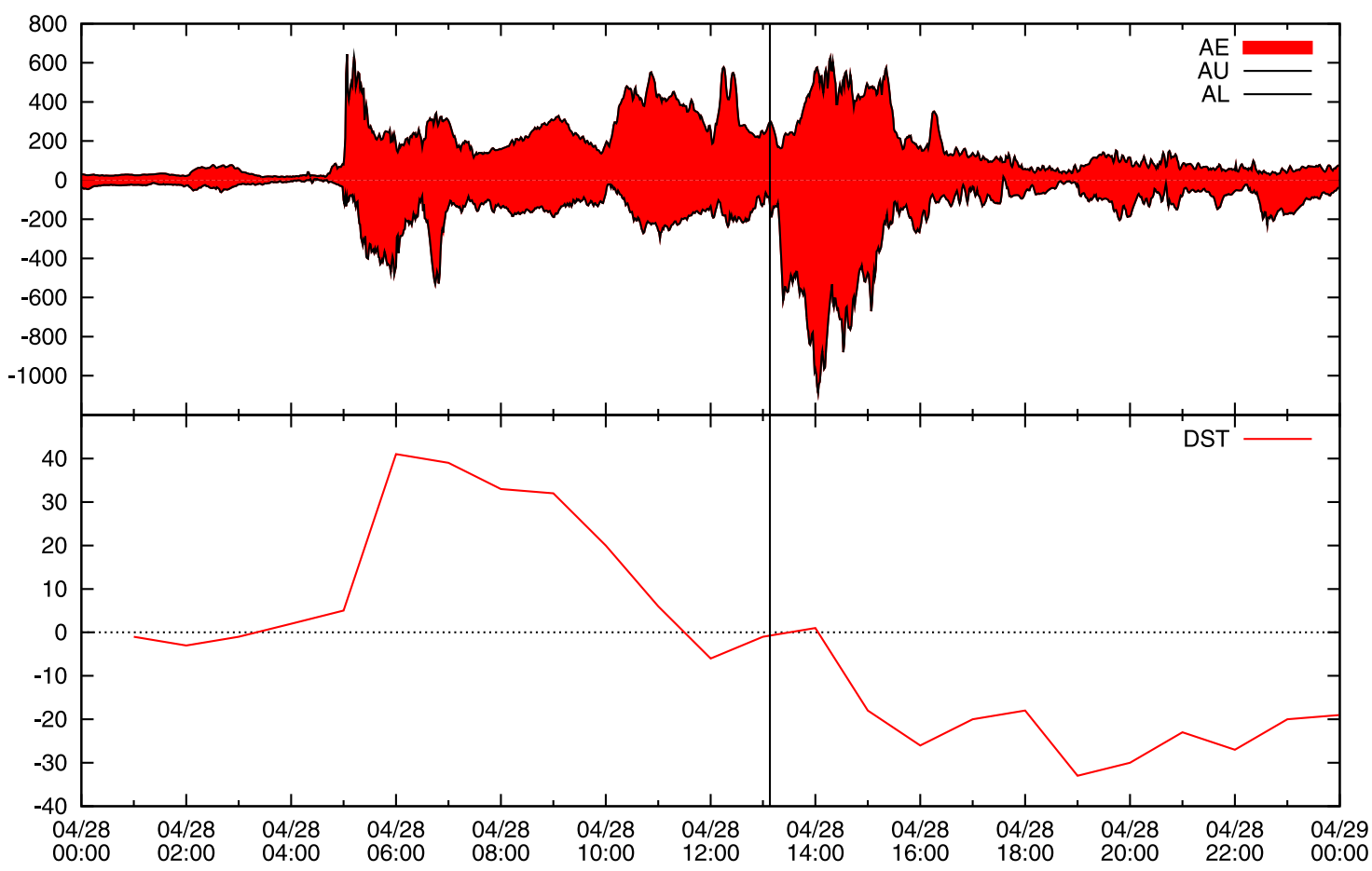

Figure 4. Provisional AE and DST indices for April 28, 2001. The vertical black line is the approximate breakup time as seen by the IMAGE spacecraft.

which clearly shows the splitting to gain more insight into what causes it.

\section{Event 2: April 28, 2001}

[20] On April 28, 2001, IMAGE recorded a substorm with an initial brightening shortly after 13:00 UT. IMAGE started collecting data on a pass over the northern hemisphere at about 11:05 UT. The first few images show significant westward propagating aurora, probably due to a previous substorm. After 11:54 UT, the images show almost no activity other than the dimly illuminated auroral oval until the breakup arc begins to brighten at the onset location at 13:07:49 UT [Frey et al., 2004]. This brightening is accompanied by a significant enhancement in the AL index, poleward expansion and westward traveling motion of the electron precipitation indicating that this is a true substorm and not a pseudo-breakup. The AU, and AL indices are plotted in Figure 4. For this event, the proton aurora also brightens significantly and expands poleward.

[21] The magnetosphere was in a reasonably disturbed state with $\mathrm{K}_{\mathrm{P}}$ between four and six and DST ranging from $40 \mathrm{nT}$ to about $-35 \mathrm{nT}$ starting at 06:00 UT until about 17:00 UT (Figure 4). However, at the time of the substorm onset the DST was around zero. The energy was loaded into the magnetosphere by an above average solar wind speed $\left(\mathrm{V}_{\mathrm{x}} \sim-650 \mathrm{~km} / \mathrm{s}\right)$ and an average number density $\left(\mathrm{n} \sim 5 \mathrm{cc}^{-3}\right)$. At the onset of the substorm, a sharp increase in the dynamic pressure was brought on by an increase in the density to approximately $8.5 \mathrm{cc}^{-3}$. The y-component of the IMF was significantly above average at $15 \mathrm{nT}$ throughout the period of interest while the $\mathrm{z}$-component varied greatly. Approximately one hour prior to the substorm, $\mathrm{B}_{\mathrm{z}}$ turned southward with a magnitude approaching $10 \mathrm{nT}$ for about half an hour.
It then turned northward with a magnitude of about $15 \mathrm{nT}$ and proceeded to decrease to about $-5 \mathrm{nT}$ at the time of onset (see Figures 5, 6 and 9).

[22] Figure 7 shows the early development of the expansion phase using the images from the WIC (electron aurora) and SI-12 (proton aurora) cameras on the IMAGE spacecraft. Two frames after the initial brightening (at 13:11:54 UT), the SI-12 instrument shows the proton precipitation peak split just eastward of the initial brightening at about 23 magnetic local time (MLT). The two proton precipitation fronts separate until the gap between them encompasses approximately two hours MLT and then remain in this configuration until after 14:30 UT. For this event, the eastward precipitation front expands westward while its eastward boundary remains fixed. The westward front continues to expand westward and its eastward boundary remains nearly co-located with the eastward boundary of the WTS.

[23] After the initial brightening of the aurora in this event, a second enhancement of the electron precipitation is evident at around 13:44 UT. This corresponds to another enhancement in the proton precipitation as well as a second drop in the AL index. Even with the very active auroral oval at that time, the proton precipitation remained split.

\subsection{Simulation and Results}

[24] For this event, the simulation domain was from $-500 \mathrm{R}_{\mathrm{E}}$ to $24 \mathrm{R}_{\mathrm{E}} \mathrm{GSE}$ in the $\mathrm{x}$-direction and from $-64 \mathrm{R}_{\mathrm{E}}$ to $64 \mathrm{R}_{\mathrm{E}}$ in $\mathrm{y}$ and $\mathrm{z}$. We used a high resolution grid with nearly 37 million cells $(616 \times 200 \times 300)$. The minimum resolution was approximately $0.2 \mathrm{R}_{\mathrm{E}}$ in $\mathrm{x}, 0.25 \mathrm{R}_{\mathrm{E}}$ in $\mathrm{y}$ and $0.16 \mathrm{R}_{\mathrm{E}}$ in $\mathrm{z}$. The high resolution region spanned the tail to a distance of approximately $40 \mathrm{R}_{\mathrm{E}}$. The grid spacing as a function of the grid axis is shown in Figure 8. 


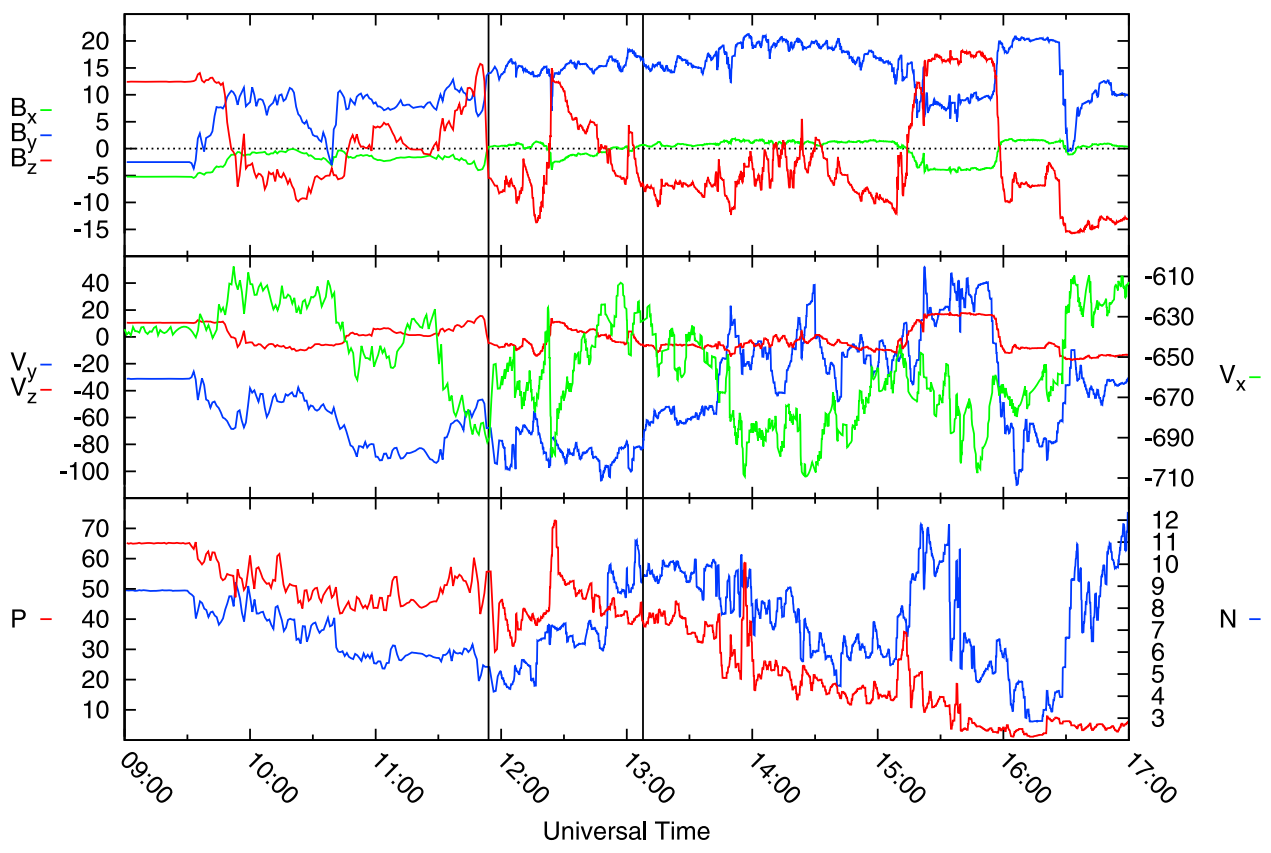

Figure 5. Solar wind input to the simulation propagated from GSE 224, 16, -24 to the sunward boundary of the simulation ( $24 \mathrm{R}_{\mathrm{E}}$ upstream). Panels show (from top to bottom) magnetic field (nT), plasma flow velocity $(\mathrm{km} / \mathrm{s})$, number density (particles per $\mathrm{cc}$ ), and thermal pressure $(\mathrm{pPa})$. The vertical lines are at 11:54:00 UT and 13:07:49 UT which are the last significant aurora seen by IMAGE SI-12 and the auroral onset respectively.

[25] Figure 9 shows the magnetic field measured at the Geotail spacecraft located in the magnetosheath. The simulation does a good job propagating the IMF $\mathrm{y}$ and $\mathrm{z}$ components into the magnetosheath, however the $\mathrm{x}$ component is not very good. This typically means that the IMF was not well ordered in sheets as assumed by the MINVAR procedure. This does not have a significant influence on the global dynamics in the magnetotail since the dayside reconnection rate is only weakly affected by the IMF $B_{x}$ as long as $\left|B_{x}\right| / B \ll 1$ because the draping of the IMF around the magnetosphere typically reduces $B_{x}$ before it interacts with the magnetosphere.

[26] The simulation reproduces the equatorward drifting of both the proton and electron precipitation maxima during the growth phase (Figure 6). Similar to the March 23, 2007 substorm, the pre-onset IB in the simulation maps to around 7-8 $\mathrm{R}_{\mathrm{E}}$. The onset occurs around 13:04 UT in the simulation. However, the typical substorm onset signatures occur over a much larger timescale in the simulation than in observations. Because of this, the chosen onset time for this event is a compromise between the enhancement of a simulated $\mathrm{AL}$ index and the most explosive brightening of the electron precipitation that occurs approximately ten minutes later. At 13:04 UT, there is slight brightening of the electron precipitation and the initial poleward expansion accompanied by westward propagation (Figure 7, column 3). There is also an enhancement of the plasma sheet temperature at that time as well which can be inferred from the increased proton energy flux in Figure 7. Also, the onset longitude is approximately correct at 13:04 UT. If the onset was placed later, the onset meridian would be displaced from the actual onset location by up to 1.5 hours MLT due to the movement of the WTS.

[27] At 13:04 UT, there is already a poleward displacement in the low latitude boundary (i.e., the IB) of the simulated proton precipitation (Figure 7). However, the scattering still persists at high latitudes resulting in pseudosplit proton precipitation (magenta arrows in Figure 7). Unlike the observations, the IB in the pseudo-split region gradually moves equatorward preventing the pseudo-splitting from becoming fully split as in the observations. This discrepancy will be discussed shortly. Presently the proton precipitation in the simulation split again. The second splitting can be seen in Figure 10. This second split clearly

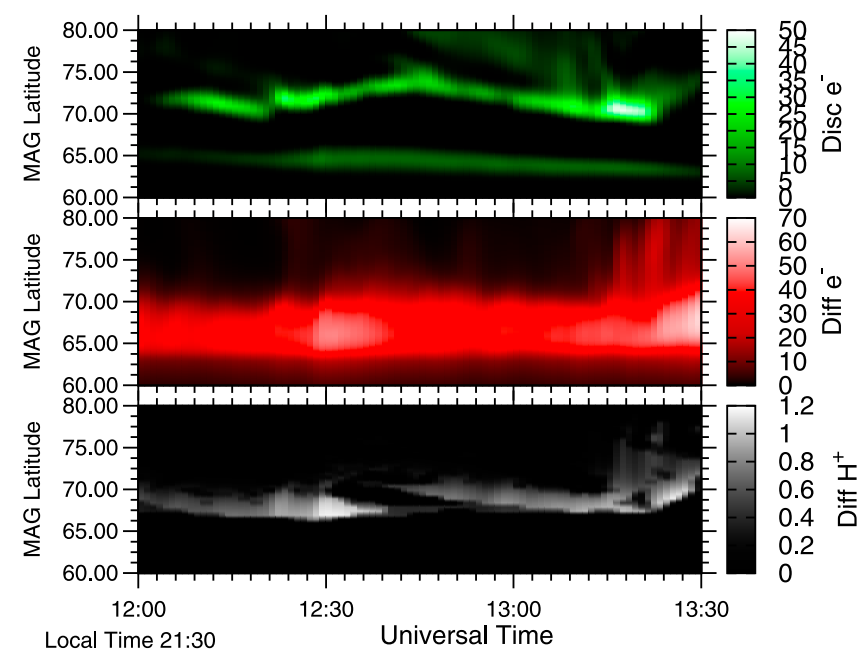

Figure 6. Simulated keogram along the 21:30 local time meridian during the growth phase of the April 28, 2001 event. The format is the same as Figure 1. The keogram reveals two auroral brightenings. The first is near 12:30 UT and the second is near 13:15 UT along this meridian. 

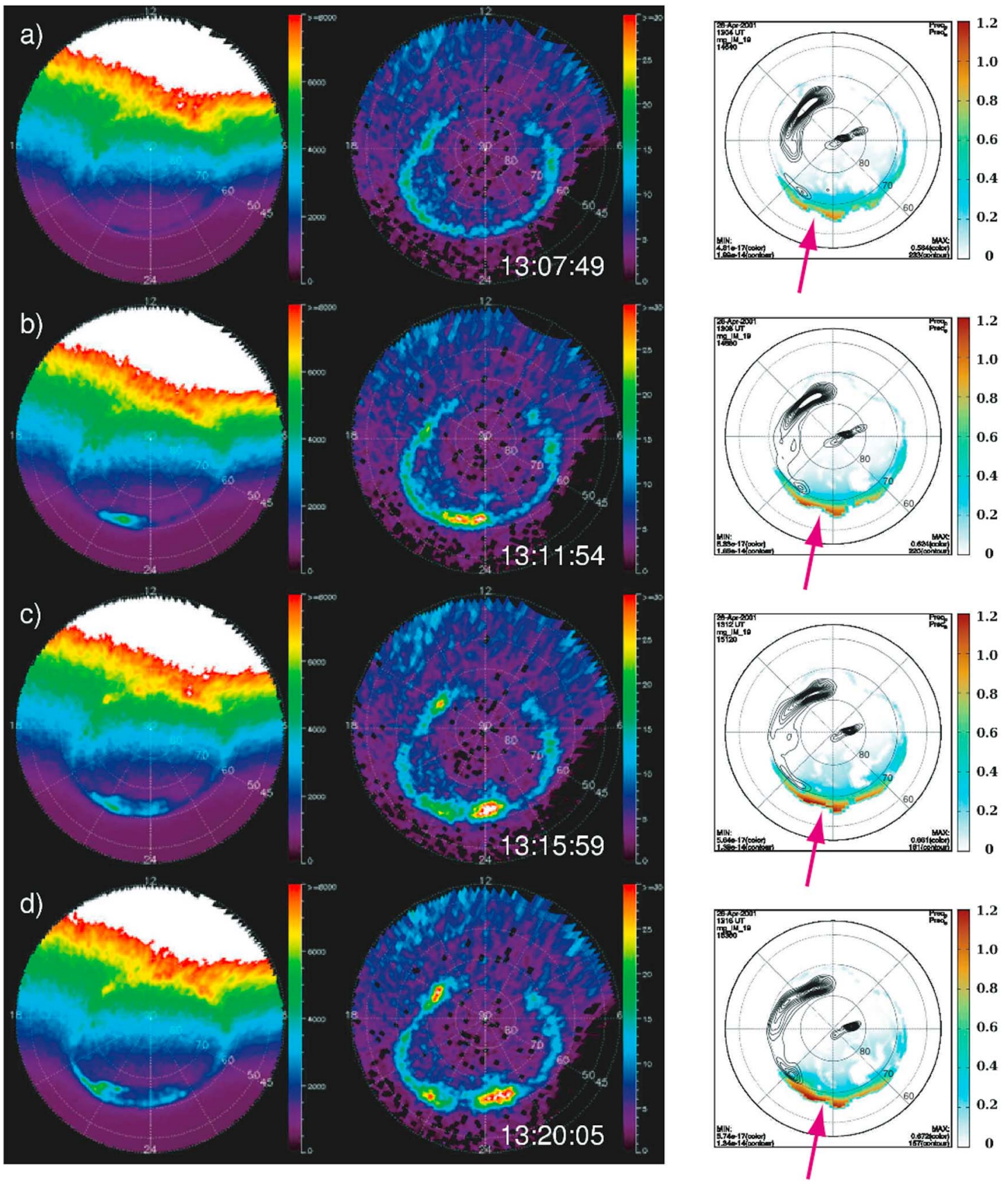

Figure 7. (a-d) Raw counts from the IMAGE WIC camera (discrete electron precipitation is shown left) and from the SI-12 camera (proton precipitation is shown in the middle) showing the evolution of the proton splitting during the first fifteen minutes of the April 28, 2001 substorm. Simulated proton precipitation with contours of discrete electron precipitation (shown right) during the late growth phase and into the early expansion phase in the simulation. The simulation reproduces some of the early splitting (magenta arrow), although it is not as dramatic as in the observations.

divides the proton aurora into two regions and remains until the end of the run. Similar to the observations, this splitting has a maximum width of approximately two hours MLT.

[28] The proton precipitation for this event is much more intense than for the March 23, 2007 substorm which is not unreasonable due to the much more extreme solar wind conditions. Additionally, the simulation produces a series of three substorms (the third substorm is the one at 13:04 UT). The first substorm happens at approximately 11:00 UT which is consistent with the observations of westward propagating aurora. However, it is a very weak substorm and it develops very slowly (probably due to the temporal proximity with the simulation initial conditions). The second substorm occurs around 12:15 UT and is most expanded and intense around 12:30 UT. This is somewhat consistent with a slight enhancement in the $\mathrm{AL}$ (and significant $\mathrm{AU}$ enhancement) at about 12:00 UT in Figure 4. However, there is not a significant auroral signature of this 


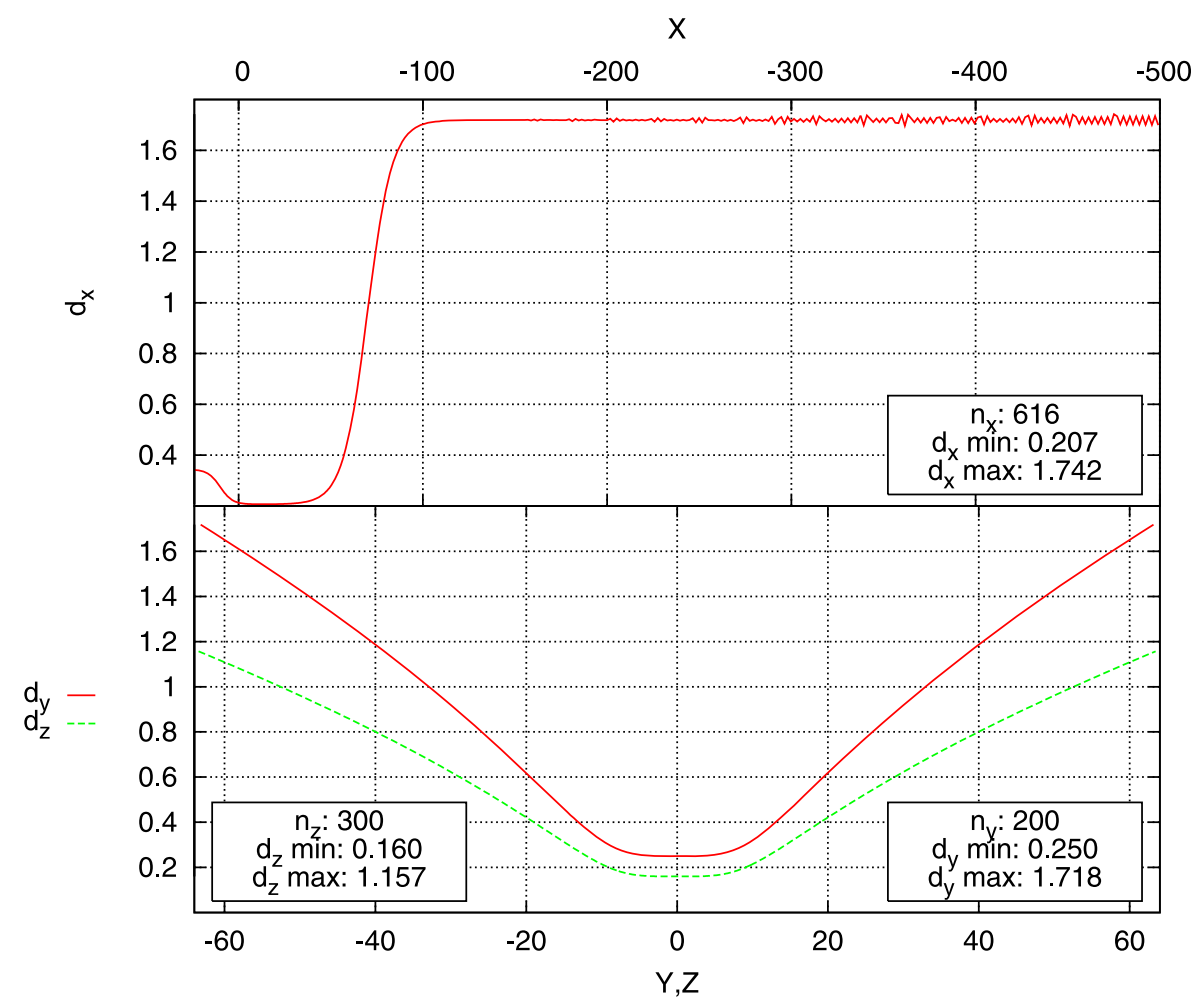

Figure 8. Grid profile used by the simulation for the April 28, 2001 event.

enhancement on the nightside in the WIC data. Therefore, the simulation seems to have magnified the magnetic disturbance into a substorm. Because of this, some of the energetic plasma remained in the geosynchronous region at the start of the 13:04 UT substorm which accounts for the high simulated energy flux at the onset. It also explains why the split region does not completely form until later in the simulation. As the substorm further energized the plasma, it quickly reached a point where the $\kappa$-scattering criteria was satisfied due to the high average gyroradii. This explains the

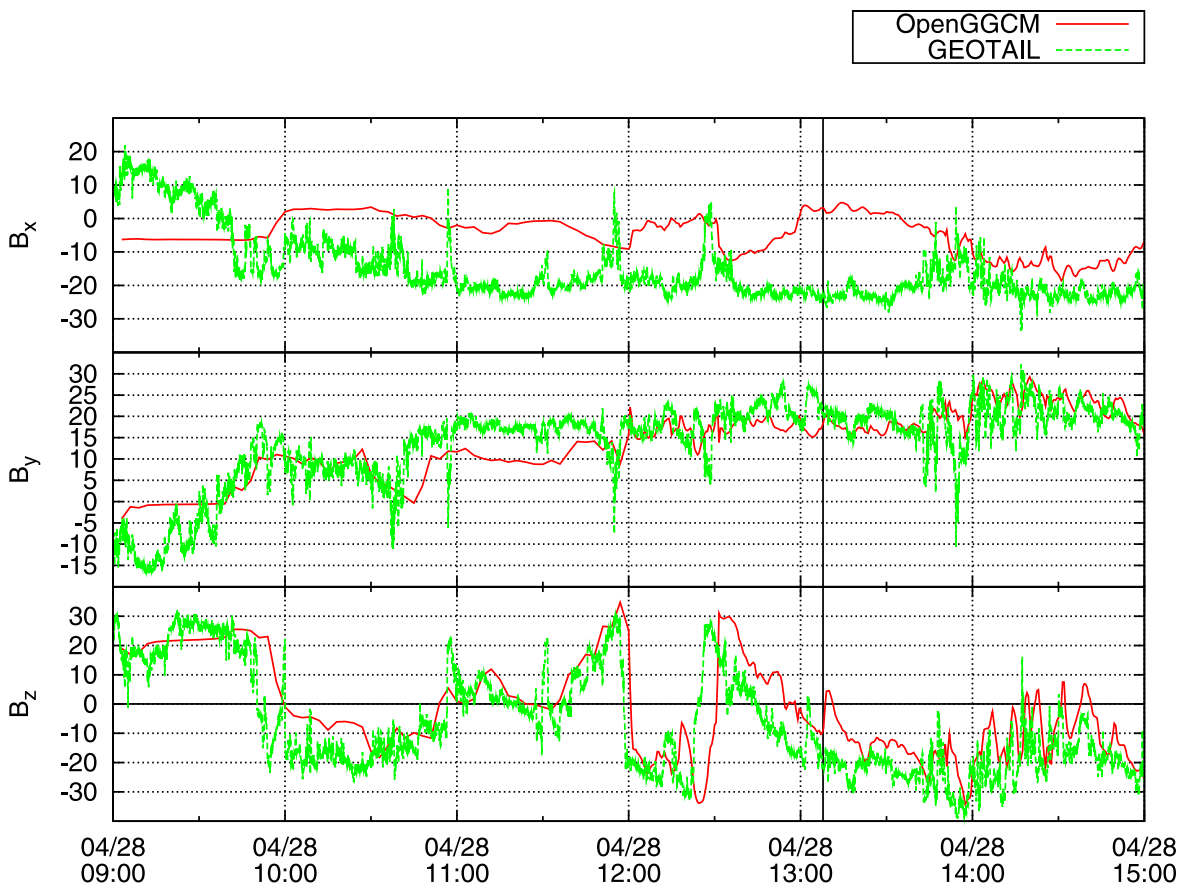

Figure 9. Comparison of simulation results (solid red line) with Geotail data (dashed green line) located in the magnetosheath at around GSE -11.5, 25.8, 1.2. The black vertical line is the approximate time of the first brightening seen by the IMAGE spacecraft. 

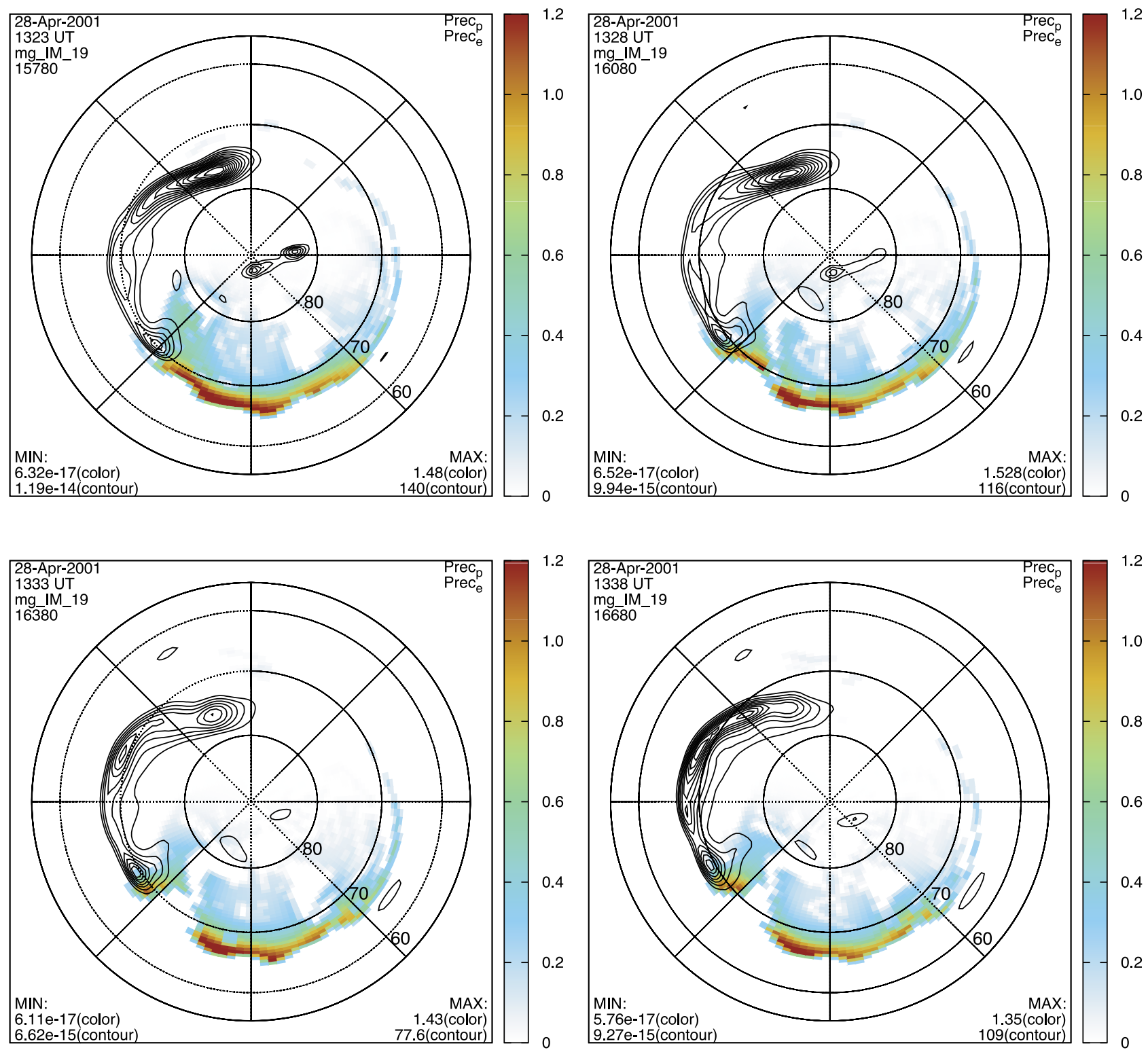

Figure 10. Simulated proton precipitation with contours of discrete electron precipitation for the April 28,2001 substorm. Local noon is at the top. The plot shows the dipolarization front at the poleward boundary of the first figure near 21 MLT. As that flux is added to the substorm current wedge, the proton scattering there is arrested resulting in two distinct proton precipitation regions.

quasi-splitting in the early expansion phase. It is also likely that the simulated magnetic field was over-stretched in the expansion phase. This is inferred because if the magnetic field was sufficiently dipolarized, the high speed flow bursts [Keiling et al., 2009; Birn et al., 2004] and pressure gradients [Lui, 1996, section 5.6, and references therein] associated with the dipolarization should have created a more significant auroral brightening.

[29] Figure 11 shows the mapped proton precipitation in the left column and $B_{z}$ in the right column for several different times around the second (complete) splitting of the proton precipitation in the simulation. The times are the same for the left and right columns and the contours of $B_{z}$ are the same to allow easier comparison of features. Similar to the March 23, 2007 substorm, this substorm also shows that the longitudinal splitting of the proton precipitation is due to the strong magnetic field with a high radius of curvature in the SCW. However, for this event, the proton precipitation does not follow the contours of $B_{z}$ the way it did for the March 23, 2007 substorm. Because of this, the reason for the splitting in this simulation requires further attention.

[30] Shortly after 13:20 UT, a new DF was driven into the magnetosphere by a strong flow channel (first panels in Figure 13 and Figure 11). It is likely that a similar flow burst produced the second brightening at 13:44 UT in the observations. This DF had low enough curvature to prevent proton scattering evidenced by the lack of proton precipitation which mapped to it. In the ionosphere, a region void of proton aurora which mapped to the DF began to extend equatorward from the poleward boundary at about 22 MLT (first 
$\mathrm{H}+$ Aurora $\left[\mathrm{mW} / \mathrm{m}^{2}\right]$

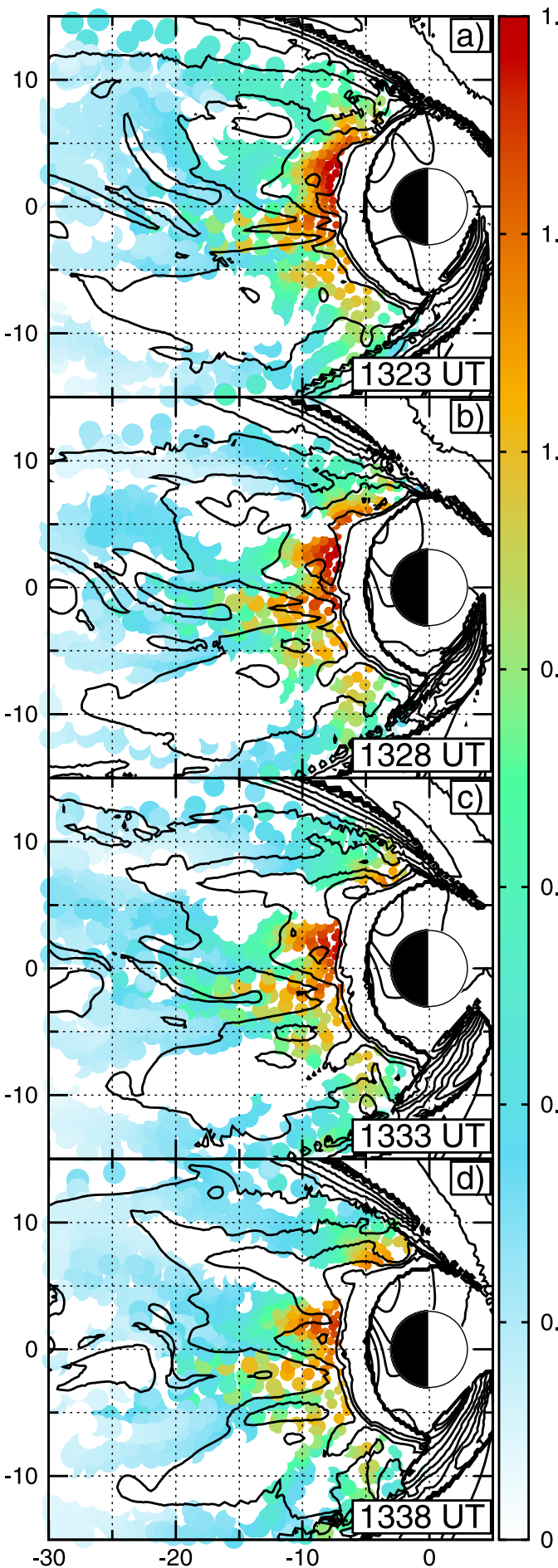

$\mathrm{B}_{\mathrm{z}}[\mathrm{nT}]$

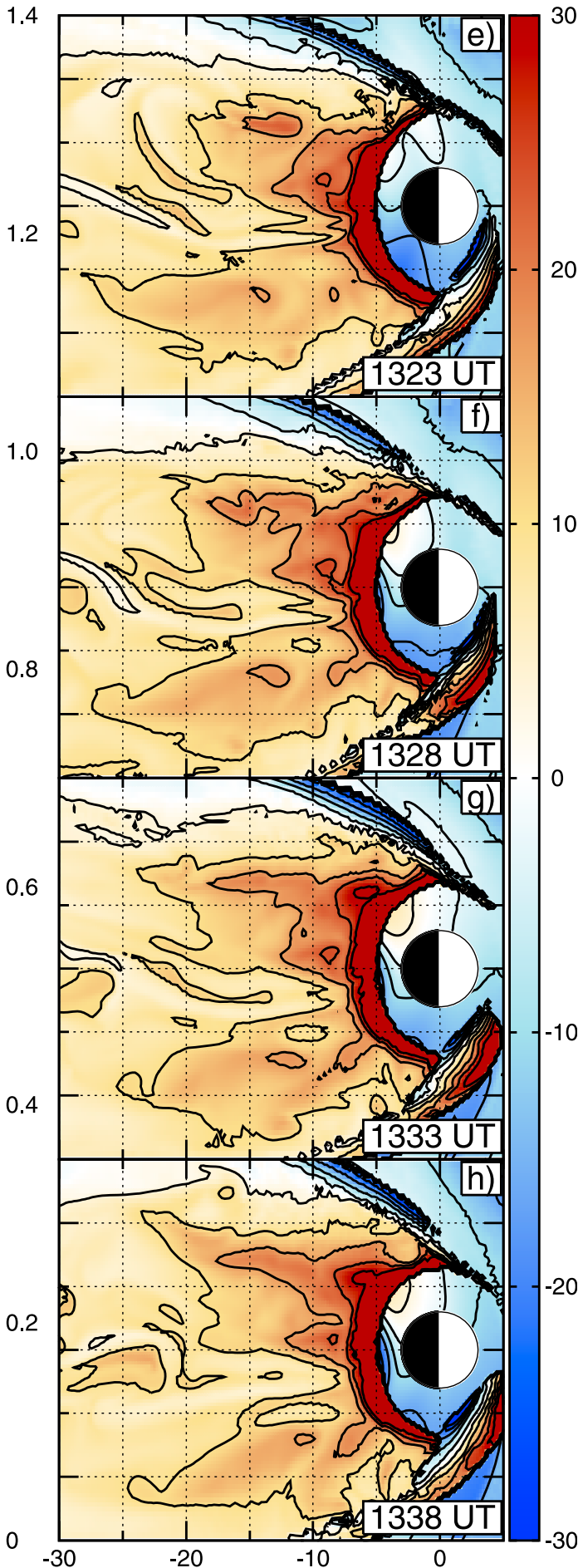

Figure 11. (a-d) Proton aurora mapped along field lines to the equatorial plane. The contours are of $B_{z}$ in the plasma sheet. (e-h) $B_{z}$ on the plane of maximum plasma $\beta$ (i.e., the central plasma sheet).

plot in Figure 10). The DF strengthened the existing SCW as they merged preventing the scattering of protons at the equatorward and poleward boundaries. Thus, the splitting is again associated with the increase in curvature and total magnetic field in the SCW. The scattering, does not follow well defined boundaries in $\mathrm{B}_{\mathrm{z}}$ for this event because of the strong azimuthal temperature dependence. Comparison with Figures 8, 9, and 11 reveals that the scattering in the eastern front is facilitated by the high temperature plasma in that region in spite of the marginally dipolarized magnetic field there.

[31] Assuming a Maxwellian distribution, the mean value of $\kappa^{2}$ at any given location is directly proportional to the 
radius of curvature and the magnitude of the magnetic field. Since dipolarization typically increases both of these quantities, the simulation always reproduces a split in the highly dipolarized regions of the SCW. However, the mean value of $\kappa^{2}$ is also inversely proportional to the square root of the temperature. In regions where the dipolarization is not as strong and the temperature is still high the scattering can continue. Therefore, the simulation demonstrates that the plasma temperature in the SCW can influence when or if the proton scattering is quenched during substorm conditions.

[32] There are a few features of the substorm that the simulation does not reliably reproduce. We have already mentioned that the plasma in the near-earth tail was overly energetic due to a previous substorm in the simulation. Also, the simulation onset latitude occurs right around 70 degrees latitude whereas the actual onset latitude was slightly below 64 degrees. This second deficiency can also be partially attributed to the earlier substorm as some of the lobe flux was probably transported to the inner magnetosphere via reconnection and enhanced convection associated with the earlier substorm. That additional flux would inflate the inner magnetosphere pushing the transition region to further radial distances (i.e., higher latitudes).

[33] Another prominent, but less import feature not reproduced by the simulation was the proton aurora extending past dusk and well into the afternoon sector. This is most likely because the scattering which caused the precipitation was most likely due to wave-particle interactions [Fuselier et al., 2004; Zhang et al., 2008; Spasojevic and Fuselier, 2009] which are not included in our model. It could also be the result of the magnetospheric compression associated with the dynamic pressure increase [Lyons et al., 2005]. However, the simulation does produce significant electron precipitation in that region which is visible above the day-glow in the IMAGE-WIC data.

[34] Despite the shortcomings of the simulation, it reproduces many of the key features of the substorm (and substorms in general) which we now summarize. In the simulation, there is an equatorward drifting of the precipitation prior to the initial auroral brightening. After the brightening, a westward traveling surge develops with the poleward expansion as is expected. The simulation produces a second increase in the proton precipitation at the same time as a second significant increase in the electron precipitation consistent with an increase in tail temperature. The observations show that the substorm had multiple brightenings (one at 13:07:49 UT and one at 13:44:37 UT) and so it is likely that the second brightening in the simulation corresponds to the second brightening in the observations. In the tail, dipolarization is observed in the near-earth region driven by bursty bulk flows. Additionally, the proton precipitation splits longitudinally in both cases. In the simulation and the data, the split region encompasses approximately sixty degrees longitude.

\section{Discussion}

[35] As mentioned briefly in the previous section, from equations (1) and (2), it is clear that:

$$
\kappa^{2} \propto \frac{R_{c}|B|}{\sqrt{T}}
$$

Using the nominal CPS temperature and a slightly lower $|B|(T=4.2 \mathrm{keV},|B|=7.5 \mathrm{nT})$ then the average gyroradius of a proton in the growth phase CPS is expected to be approximately $0.2 \mathrm{R}_{\mathrm{E}}$. The current sheet thickness is often on the order of $2000 \mathrm{~km}$ or less [Baumjohann et al., 2007; Zhou et al., 2009]. Using these parameters, the growth phase $\kappa$ is $\sim 1.2$.

[36] At the start of the expansion phase, if the field inside the SCW dipolarizes completely, it can be shown from equation (3) that in the equatorial plane:

$$
R_{c}=\frac{R}{2}
$$

where $R$ is the radial distance to the Earth's center. Thus, $R_{c}$ can increase by a factor of $\sim 10$ or more at $8 \mathrm{R}_{\mathrm{E}}$. Additionally, the magnitude of the magnetic field increases with the dipolarization. In the equatorial plane, if the growth phase $|B|$ was $\sim 7.5 \mathrm{nT}$ and the field dipolarized completely $\left(|B|=\frac{M}{R^{3}}\right)$, the field strength would increase by a factor of $\sim 8$. These two factors would yield an increase in $\kappa$ by a factor of nearly 10 (at $8 \mathrm{R}_{\mathrm{E}}$ ) if the field dipolarized completely. Of course, $\kappa$ still depends on $T^{-1 / 4}$. It is common for the temperature to increase during a substorm by a factor of $\sim 4$ [Baumjohann, 1991]. Including this temperature increase still yields an increase in $\kappa$ by a factor of $\sim 7$ at $8 \mathrm{R}_{\mathrm{E}}$. More concretely, using equations (2) and (7), the average value for $\kappa$ in the equatorial plane of a dipole field is:

$$
\kappa_{\text {dipole }}=\left(\frac{M}{R^{2} \sqrt{8 m_{i} k_{b} T_{i}}}\right)^{\frac{1}{2}}
$$

where $\mathrm{M}$ is the dipole moment (30.4 $\mu \mathrm{T} \cdot \mathrm{R}_{\mathrm{E}}^{3}$ for Earth). Figure 12 shows equation (8) for a few different plasma temperatures. Clearly, in a completely dipolar field, the scattering should be quenched. However, if the dipolarization is not so complete (if $|B|$ and $R_{c}$ increase by only a factor of 3.5 for example), but the plasma temperature still increases by a factor of $4, \kappa$ will only increase by a factor of 2.5 which results in an average $\kappa$ after dipolarization of $\sim 3$ which is right at the edge of the scattering threshold (equation (1)).

[37] In both of the simulations presented here, the edges of the split region in the proton precipitation maps to the dipolarized SCW. However, in the April 28, 2001 event, there seems to be two levels of dipolarization. The inner, highly dipolarized region centered near $\mathrm{x}=-6, \mathrm{y}=6$ contains the majority of the SCW flux. There is also a less dipolarized region which extends from about $\mathrm{y}=0$ to $\mathrm{y}=8$ (Figures $11 \mathrm{c}$ and $11 \mathrm{~g}$ ) which forms a secondary (less dipolarized) SCW that encompasses the first. The proton precipitation from the outer region is influenced by the high temperature plasma on its eastern flank which overcomes the lower curvature to produce scattering (Figure 13).

[38] This suggests that regions of high temperature plasma and less stretched but still non-dipolar field can exist during substorm conditions. If that energization profile existed globally and the field did not dipolarize as significantly, there would be no splitting of the proton aurora which is also consistent with the speculation by Gilson et al. [2011]. It is expected that substorms with that configuration would produce weaker field-aligned currents due to smaller pressure gradients and smaller flow bursts expected with the weaker 


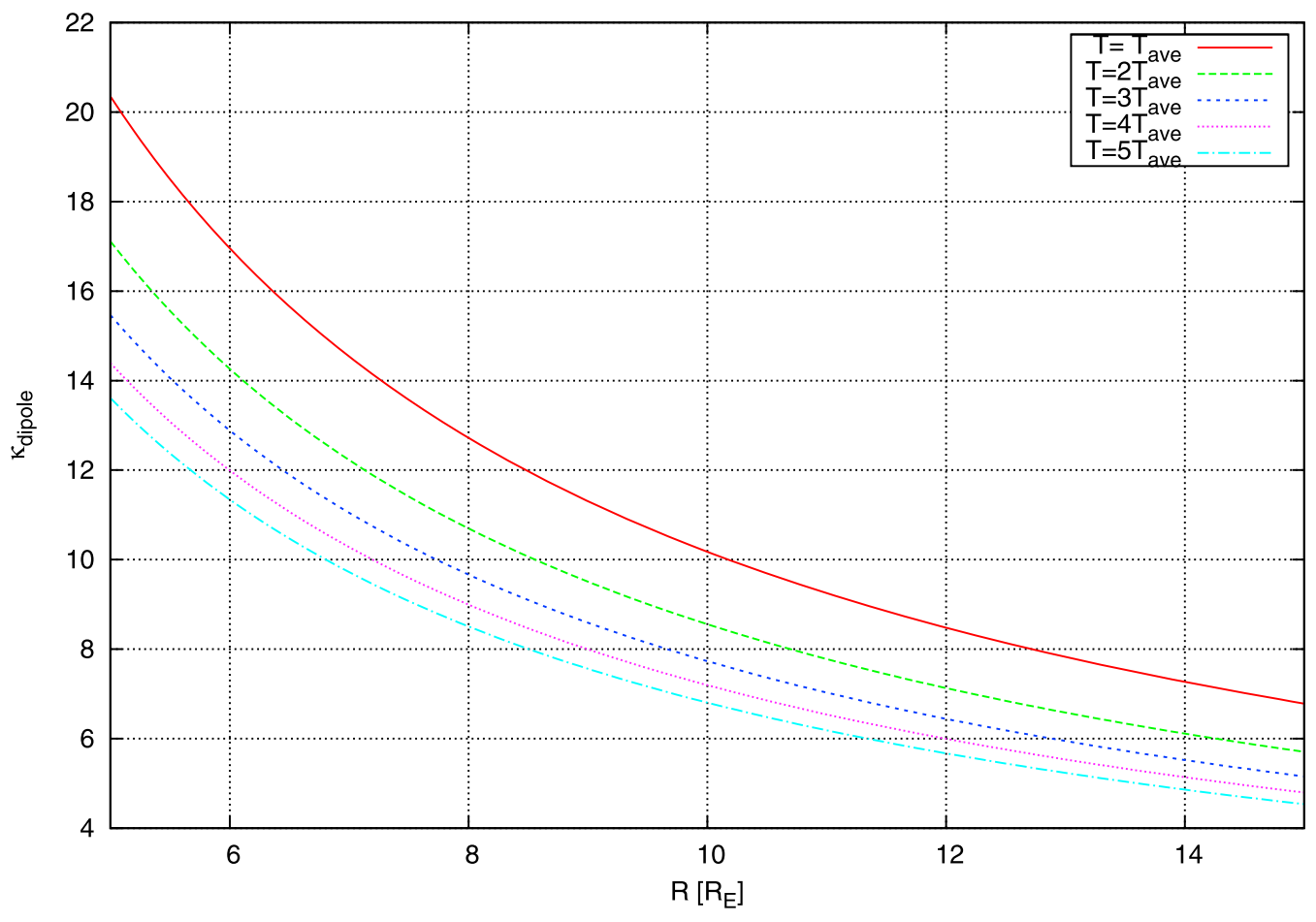

Figure 12. Average values for $\kappa$ in the equatorial plane of Earth's dipole magnetic field as a function of distance. For a large range of temperatures, $\kappa$ remains above 3 indicating that it is very difficult to scatter protons into the loss cone in a dipolar geometry.

dipolarization. From this perspective, it is reasonable that the proton aurora is less likely to split during weaker substorms.

[39] Our model has a few shortcomings that must be addressed. The MHD model used in this study cannot accurately capture all of the physics associated with substorm particle injections [e.g., Birn et al., 1997; Spanswick et al., 2009]. As these injections tend to accelerate the high energy portion of the distribution, they will also make that portion of the distribution more likely to scatter and precipitate while the lower energy (i.e., thermal) portion of the distribution is not available to precipitate. This effect would not be picked up by our model and would also decrease the likelihood of observing (complete) splitting for a particular event. Additionally, magnetotail particle distributions observed are typically more like kappa distributions than Maxwellian [e.g., Haaland et al., 2010]. Since kappa distributions have a larger particle flux in the high energy portion of the spectrum our calculations probably underestimate the energy flux into ionosphere from neglecting those higher energy particles.

[40] Even in a thermal distribution of particles, our model has some shortcomings. It assumes that the scattering from a region of space is determined only by the average gyroradius of the distribution. For a given field geometry, this produces a critical temperature threshold for turning on and off the scattering along that flux tube. In reality, a distribution just below the critical temperature and a distribution just above the critical temperature will likely produce similar precipitation. This assumption probably leads to an over estimation of the flux into the magnetosphere especially in regions where the temperature is close to the critical temperature which probably partially offsets the error from assuming a
Maxwellian particle distribution instead of a Kappa distribution. In any event, due to these simplifying assumptions, the calculated energy flux into the ionosphere is only expected to be qualitatively similar to observations.

[41] The single fluid MHD used for the simulations presented here also does not resolve the physics associated with gradient and curvature drifting of ions in the inner magnetosphere. This mechanism is often used to explain a dawndusk asymmetry in the proton precipitation. However, Newell et al. [2009] shows that during moderately strong solar wind driving, the asymmetry is not consistent with the conventional wisdom. Because of this, it is unclear the role that the high energy drifting ions play in the precipitation during times of increased geomagnetic activity. In any event, those ions are not reproduced in the MHD model and any precipitation resulting from those ions is not reproduced in our simulations.

[42] Finally, the model neglects transit time from the magnetosphere to the ionosphere. Since the bounce time for a thermal proton is on the order of a minute, this can introduce some additional timing ambiguity. However, this timing ambiguity is generally less than the ambiguity already present when trying to interpret the MHD simulation results and is therefore probably not significant.

\section{Summary and Conclusion}

[43] We have presented simulations of the global nightside proton precipitation using the output of a global MHD simulation and the field line curvature model to determine the loss cone filling. The simulation reproduces the global 
$\mathrm{T}[\mathrm{keV}]$

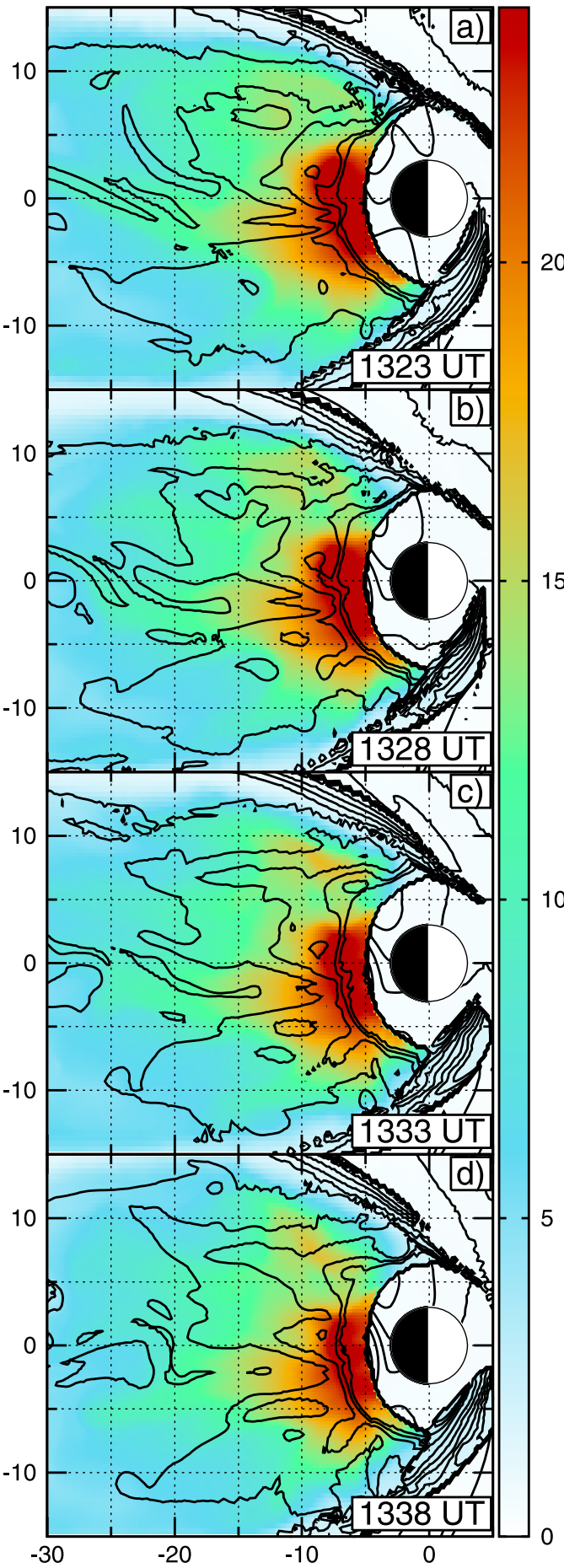

$\mathrm{Vx}[\mathrm{km} / \mathrm{s}]$

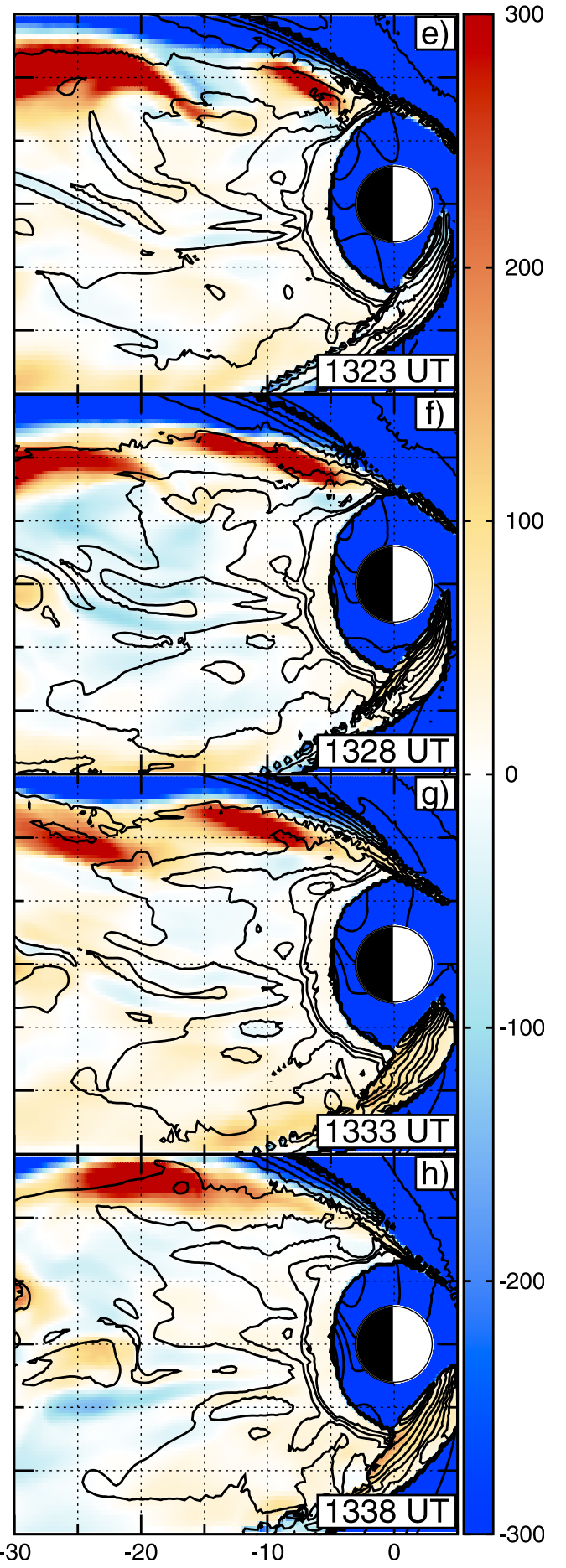

Figure 13. $(a-d)$ Temperature and $(e-h) V_{x}$ on the central plasma sheet. The contours are the same as in Figure 11. A flow channel opens and drives a dipolarization front into the inner magnetosphere where the flux piles up and forms the current wedge. The high temperature region on the eastward flank of the current wedge causes the scattering criteria to be satisfied even though the field is marginally dipolarized there.

features of the observed proton aurora during substorms (equatorward motion during the growth phase, intensification and slight poleward expansion at onset) and the general ordering of precipitation boundaries presented by Mende [2003]. The simulation supports the interpretation of
Sergeev et al. [1983] that the nightside proton precipitation is dominated by field line curvature scattering of CPS protons into the loss cone since the simulation captures the global features of the proton aurora without including waveparticle interactions. While this idea is not new, we have 
calculated the field geometry and average gyroradius selfconsistently instead of assuming field geometries and/or particle distributions as done previously [Sergeev and Tsyganenko, 1982; Sergeev et al., 1983; Delcourt and Martin, 1994; Delcourt et al., 1996]. Also, we have compared our simulation with data from global proton auroral images and shown that it reproduces the several key features near the substorm onset.

[44] Of particular interest to this study is the longitudinal splitting reported by Gilson et al. [2011]. The simulation supports their interpretation that the splitting is mapped into the SCW where the dipolarized magnetic field prevents the scattering criteria (equation (2)) from being satisfied. However, the simulation for the April 28, 2001 event shows that even where the magnetic field is moderately dipolarized, significant temperature (gyroradius) enhancements can still cause precipitation. The simulation results suggest that the split region always maps into the $\mathrm{SCW}$, but that the edges of the split region may not correspond to the edges of the SCW (i.e., the SCW may be larger than the region where the split maps). In fact, the simulation implies that the edge of the SCW is not necessarily an easy boundary to define since there can be a highly dipolarized region embedded in a moderately dipolarized region. This and the energization from substorm particle injections are most likely the reasons why not all the substorms in the statistical study by Gilson et al. [2011] exhibited clear proton auroral splitting. The same reasoning can be used to explain why the time delay between substorm onset and proton auroral splitting was commonly longer than the proton bounce time in the study by Gilson et al. [2011]. Finally, in the simulation, the location of the IB maps to $\sim 7-8 \mathrm{R}_{\mathrm{E}}$ in the magnetotail for both substorms examined.

[45] In the future studies, we will run the global MHD model with a coupled inner magnetosphere model. That should improve our mapping and also capture the gradient curvature drift effects and more of the particle injection physics that the global MHD model lacked in this study. The improved mapping and boundary conditions in the inner magnetosphere should produce a more reliable estimate for the mapping of the IB. Also, since the intensity of the simulated proton precipitation depends on the plasma temperature, the simulated proton precipitation should enable us to develop new tools to use when studying particle injections in the coupled global MHD model. Thus, we would like to understand the source and location of the peak in proton auroral brightness in the magnetosphere.

[46] Using the inner magnetosphere model of Fok et al. [2001], pitch angle distributions will also be able to be simulated. With this, temperature anisotropies and growth of Electro-Magnetic Ion Cyclotron (EMIC) waves could be modeled [Blum et al., 2009]. Using this, it should be possible to expand the above described proton aurora code to include the precipitation from wave scattering by EMIC waves.

[47] Acknowledgments. This work was supported by grant NAS502099 from NASA. Development of the OpenGGCM is supported by grant ATM-0639658 from the NSF. The authors would also like to acknowledge CDAweb for providing plotting utilities and hosting IMAGE and other satellite data used in this study. Thanks to Stephen Mende, Harald Frey and the IMAGE team for providing data and analysis tools. Also, the authors would like to thank the reviewers for their careful attention to detail and other comments on this paper.

[48] Robert Lysak thanks the reviewers for their assistance in evaluating this paper.

\section{References}

Angelopoulos, V., et al. (2008), First results from the THEMIS mission, Space Sci. Rev., 141(1-4), 453-476, doi:10.1007/s11214-008-9378-4.

Ashour-Abdalla, M., and R. M. Thorne (1978), Toward a unified view of diffuse auroral precipitation, J. Geophys. Res., 83(A10), 4755-4766.

Baumjohann, W. (1991), Heating and fast flows in the near-Earth tail, in Magnetospheric Substorms, Geophys. Monogr. Ser., vol. 64, edited by J. R. Kan et al., pp. 141-145, AGU, Washington, D. C., doi:10.1029/ GM064p0141.

Baumjohann, W., et al. (2007), Dynamics of thin current sheets: Cluster observations, Ann. Geophys., 25, 1365-1389.

Birn, J., M. F. Thomsen, J. E. Borovsky, G. D. Reeves, D. J. McComas, and R. D. Belian (1997), Characteristic plasma properties during dispersionless substorm injections at geosynchronous orbit, J. Geophys. Res., 102(A2), 2309-2324, doi:10.1029/96JA02870.

Birn, J., J. Raeder, Y. L. Wang, R. A. Wolf, and M. Hesse (2004), On the propagation of bubbles in the geomagnetic tail, Ann. Geophys., 22, 1773-1786.

Blockx, C., J.-C. Gérard, M. Meurant, B. Hubert, and V. Coumans (2005) Far ultraviolet remote sensing of the isotropy boundary and magnetotail stretching, J. Geophys. Res., 110, A11215, doi:10.1029/2005JA011103.

Blum, L. W., E. A. MacDonald, S. P. Gary, M. F. Thomsen, and H. E. Spence (2009), Ion observations from geosynchronous orbit as a proxy for ion cyclotron wave growth during storm times, J. Geophys. Res., 114, A10214, doi:10.1029/2009JA014396.

Büchner, J., and L. M. Zelenyi (1987), Chaotization of the electron motion as the cause of an internal magnetotail instability and substorm onset, J. Geophys. Res., 92(A12), 13,456-13,466.

Creutzberg, F., R. L. Gattinger, F. R. Harris, S. Wozniak, and A. V. Jones (1988), Auroral studies with a chain of meridian scanning photometers: 2. Mean distributions of proton and electron aurora as a function of magnetic activity, J. Geophys. Res., 93(A12), 14,591-14,601, doi:10.1029/ JA93iA12p14591.

Deehr, C., and D. Lummerzheim (2001), Ground-based optical observations of hydrogen emission, J. Geophys. Res., 106(A1), 33-44.

Delcourt, D. C., and R. F. Martin (1994), Application of the centrifugal impulse model to particle motion in the near-Earth magnetotail, J. Geophys. Res., 99(A12), 23,583-23,590, doi:10.1029/94JA01845.

Delcourt, D. C., J.-A. Sauvaud, R. F. Martin, and T. E. Moore (1996), On the nonadiabatic precipitation of ions from the near-Earth plasma sheet, J. Geophys. Res., 101(A8), 17,409-17,418.

Donovan, E. F., B. J. Jackel, R. J. Strangeway, and D. M. Klumpar (2003a), Energy dependence of the latitude of the $1-25 \mathrm{KeV}$ ion isotropy boundary, in Sodankylä Geophysical Observatory Publications, vol. 92, pp. 11-14, Oulu Univ. Press, Oulu, Finland.

Donovan, E. F., B. J. Jackel, I. Voronkov, T. Sotirelis, F. Creutzberg, and N. A. Nicholson (2003b), Ground-based optical determination of the b2i boundary: A basis for an optical MT-index, J. Geophys. Res., 108(A3), 1115, doi:10.1029/2001JA009198.

Eather, R. H. (1967), Auroral proton precipitation and hydrogen emissions, Rev. Geophys., 5(3), 207-285, doi:10.1029/RG005i003p00207.

Fok, M., R. A. Wolf, R. W. Spiro, and T. E. Moore (2001), Comprehensive computational model of Earth's ring current, J. Geophys. Res., 106(A5), $8417-8424$.

Frey, H. U., S. B. Mende, and V. Angelopoulos (2004), Substorm onset observations by IMAGE-FUV, J. Geophys. Res., 109, A10304, doi:10.1029/2004JA010607.

Fuller-Rowell, T. J., D. Rees, S. Quegan, R. J. Moffett, M. V. Codrescu, and G. H. Millward (1996), A coupled thermosphere-ionosphere model (CTIM), in STEP Report, edited by R. W. Schunk, p. 217, Sci. Comm. on Sol. Terr. Phys., Boulder, Colo.

Fuselier, S. A., S. P. Gary, M. F. Thomsen, E. S. Clafin, B. Hubert, B. R. Sandel, and T. J. Immel (2004), Generation of transient dayside subauroral proton precipitation, J. Geophys. Res., 109, A12227, doi:10.1029/ 2004JA010393.

Gilson, M. L., J. Raeder, E. F. Donovan, Y. S. Ge, and S. B. Mende (2011), Statistics of the longitudinal splitting of proton aurora during substorms, J. Geophys. Res., 116, A08226, doi:10.1029/2011JA016640.

Gvozdevsky, B. B., and V. A. Sergeev (1996), MT-index-A possible new index to characterize the configuration of the magnetotail, $A d v$. Space Res., 18(8), 51-54, doi:10.1016/0273-1177(95)00995-7.

Haaland, S., E. A. Kronberg, P. W. Daly, M. Fränz, L. Degener, E. Georgescu, and I. Dandouras (2010), Spectral characteristics of protons in the Earth's plasmasheet: Statistical results from Cluster CIS and RAPID, Ann. Geophys., 28, 1483-1498, doi:10.5194/angeo-28-1483-2010.

Keiling, A., et al. (2008), Correlation of substorm injections, auroral modulations and ground Pi2, Geophys. Res. Lett., 35, L17S22, doi:10.1029/ 2008GL033969. 
Keiling, A., et al. (2009), Substorm current wedge driven by plasma flow vortices: THEMIS observations, J. Geophys. Res., 114, A00C22, doi:10.1029/2009JA014114

Kistler, L. M., et al. (1998), FAST/TEAMS observations of charge exchange signatures in ions mirroring at low altitudes, Geophys. Res. Lett., 25(12), 2085-2088.

Lessard, M. R., W. Lotko, J. LaBelle, W. Peria, C. W. Carlson, F. Creutzberg, and D. D. Wallis (2007), Ground and satellite observations of the evolution of growth phase auroral arcs, J. Geophys. Res., 112, A09304, doi:10.1029/ 2006JA011794.

Lessard, M. R., et al. (2009), PENGUIn multi-instrument observations of dayside high-latitude injections during the 23 March 2007 substorm, J. Geophys. Res., 114, A00C11, doi:10.1029/2008JA013507.

Liu, W. W., E. F. Donovan, J. Liang, I. Voronkov, E. Spanswick, P. T. Jayachandran, B. J. Jackel, and M. Meurant (2007), On the equatorward motion and fading of proton aurora during substorm growth phase, J. Geophys. Res., 112, A10217, doi:10.1029/2007JA012495.

Lui, A. T. Y. (1996), Current disruption in the Earth's magnetosphere: Observations and models, J. Geophys. Res., 101(A6), 13,067-13,088, doi:10.1029/96JA00079.

Lyons, L. R., D. Lee, C. Wang, and S. B. Mende (2005), Global auroral responses to abrupt solar wind changes: Dynamic pressure, substorm, and null events, J. Geophys. Res., 110, A08208, doi:10.1029/2005JA011089.

Mende, S. B. (2003), Statistical behavior of proton and electron auroras during substorms, J. Geophys. Res., 108(A9), 1339, doi:10.1029/ 2002JA009751.

Mende, S. B., et al. (2000a), Far ultraviolet imaging from the IMAGE spacecraft. 2. Wideband FUV imaging, Space Sci. Rev., 91(1), 271-285.

Mende, S. B., et al. (2000b), Far ultraviolet imaging from the IMAGE spacecraft. 1. System design, Space Sci. Rev., 91(1), 243-270.

Mende, S. B., et al. (2000c), Far ultraviolet imaging from the IMAGE spacecraft. 3. Spectral imaging of Lyman-alpha and OI $135.6 \mathrm{~nm}$, Space Sci. Rev., 91(1), 287-318.

Meurant, M., J.-C. Gérard, C. Blockx, E. Spanswick, E. F. Donovan, B. Hubert, V. Coumans, and M. Connors (2007), EL - a possible indicator to monitor the magnetic field stretching at global scale during substorm expansive phase: Statistical study, J. Geophys. Res., 112, A05222, doi:10.1029/2006JA012126.

Newell, P. T., Y. I. Feldstein, Y. I. Galperin, and C.-I. Meng (1996), Morphology of nightside precipitation, J. Geophys. Res., 101(A5) 10,737-10,748, doi:10.1029/95JA03516.

Newell, P. T., V. A. Sergeev, G. R. Bikkuzina, and S. Wing (1998), Characterizing the state of the magnetosphere: Testing the ion precipitation maxima latitude (b2i) and the ion isotropy boundary, J. Geophys. Res., 103(A3), 4739-4745, doi:10.1029/97JA03622.

Newell, P. T., T. Sotirelis, and S. Wing (2009), Diffuse, monoenergetic, and broadband aurora: The global precipitation budget, J. Geophys. Res., 114, A09207, doi:10.1029/2009JA014326.

Nishimura, Y., et al. (2010), Identifying the driver of pulsating aurora, Science, 330(6000), 81-84, doi:10.1126/science.1193186.

Raeder, J. (2003), Global magnetohydrodynamics-A tutorial, in Space Plasma Simulation, vol. 615, pp. 212-246, Springer, Berlin.

Raeder, J., Y. Wang, and T. J. Fuller-Rowell (2001), Geomagnetic storm simulation with a coupled magnetosphere-ionosphere-thermosphere model, in Space Weather, Geophys. Monogr. Ser., vol. 125, edited by
P. Song, H. J. Singer, and G. L. Siscoe, pp. 377-384, AGU, Washington, D. C., doi:10.1029/GM125p0377.

Raeder, J., D. Larson, W. Li, L. Kepko, and T. J. Fuller-Rowell (2008), OpenGGCM simulations for the THEMIS mission, Space Sci. Rev., 141(1-4), 535-555, doi:10.1007/s11214-008-9421-5.

Raeder, J., P. Zhu, Y. S. Ge, and G. L. Siscoe (2010), Open geospace general circulation model simulation of a substorm: Axial tail instability and ballooning mode preceding substorm onset, J. Geophys. Res., 115, A00I16, doi:10.1029/2010JA015876.

Runov, A., V. Angelopoulos, N. Ganushkina, R. Nakamura, J. P. McFadden, D. Larson, I. Dandouras, K.-H. Glassmeier, and C. Carr (2008), Multipoint observations of the inner boundary of the plasma sheet during geomagnetic disturbances, Geophys. Res. Lett., 35, L17S23, doi:10.1029/ 2008GL033982.

Russell, C. T., et al. (2000), The interplanetary shock of September 24, 1998: Arrival at Earth, J. Geophys. Res., 105(A11), 25,143-25,154, doi:10.1029/2000JA900070.

Sergeev, V. A., and N. A. Tsyganenko (1982), Energetic particle losses and trapping boundaries as deduced from calculations with a realistic magnetic field model, Planet. Space Sci., 30(10), 999-1006, doi:10.1016/ 0032-0633(82)90149-0.

Sergeev, V. A., E. Sazhina, N. A. Tsyganenko, J. Lundblad, and F. Soraas (1983), Pitch-angle scattering of energetic protons in the magnetotail current sheet as the dominant source of their isotropic precipitation into the nightside ionosphere, Planet. Space Sci., 31(10), 1147-1155, doi:10.1016/0032-0633(83)90103-4.

Sergeev, V. A., M. Malkov, and K. Mursula (1993), Testing the isotropic boundary algorithm method to evaluate the magnetic field configuration in the tail, J. Geophys. Res., 98(A5), 7609-7620.

Sergeev, V. A., Y. Nishimura, M. Kubyshkina, V. Angelopoulos, R. Nakamura, and H. Singer (2012), Magnetospheric location of the equatorward prebreakup arc, J. Geophys. Res., 117, A01212, doi:10.1029/2011JA017154

Sonnerup, B. U. O., and L. J. Cahill (1967), Magnetopause structure and attitude from Explorer 12 observations, J. Geophys. Res., 72, 171-183.

Spanswick, E., E. F. Donovan, W. W. Liu, J. Liang, J. B. Blake, G. D. Reeves, R. Friedel, B. J. Jackel, C. Cully, and A. T. Weatherwax (2009), Global observations of substorm injection region evolution: 27 August 2001, Ann. Geophys., 27(2002), 2019-2025.

Spasojevic, M., and S. A. Fuselier (2009), Temporal evolution of proton precipitation associated with the plasmaspheric plume, J. Geophys. Res., 114, A12201, doi:10.1029/2009JA014530.

Tsyganenko, N. A. (1989), A magnetospheric magnetic field model with a warped tail current sheet, Planet. Space Sci., 37, 5-20.

Tsyganenko, N. A. (2002a), A model of the near magnetosphere with a dawn-dusk asymmetry: 1. Mathematical structure, J. Geophys. Res., 107(A8), 1179, doi:10.1029/2001JA000219.

Tsyganenko, N. A. (2002b), A model of the near magnetosphere with a dawn-dusk asymmetry: 2. Parameterization and fitting to observations, J. Geophys. Res., 107(A8), 1176, doi:10.1029/2001JA000220.

Zhang, Y., L. J. Paxton, and Y. Zheng (2008), Interplanetary shock induced ring current auroras, J. Geophys. Res., 113, A01212, doi:10.1029/ 2007JA012554.

Zhou, X.-Z., et al. (2009), Thin current sheet in the substorm late growth phase: Modeling of THEMIS observations, J. Geophys. Res., 114 A03223, doi:10.1029/2008JA013777. 